\title{
Metabolites of intestinal microflora upregulate microRNA-200c-3p expression level to suppress airway epithelial inflammation via the IL6ST/JNK/STAT3 signaling pathway
}

\author{
LINLIANG HONG, HUANHUAN HUANG and BIN WU
}

\author{
Department of Pediatrics, The First Affiliated Hospital of Fujian Medical University, Fuzhou, Fujian 350005, P.R. China
}

Received June 28, 2020; Accepted March 8, 2021

DOI: $10.3892 / \mathrm{etm} .2021 .10431$

\begin{abstract}
Intestinal microfloras are involved in various types of cancer; however, there is a limited amount of research into the involvement of metabolites of intestinal microflora (MIM) in asthmatic airway epithelial cells (AECs). The present study was designed to reveal the functions and mechanisms of MIM in the asthmatic inflammation of AECs. House dust mite (HDM)-induced asthma cell models were established and treated with mouse MIM. A MTT assay was used to investigate AEC viability, while reverse transcription-quantitative PCR and western blot analysis were used to measure the expression levels of miR-200c-3p, IL6ST, JNK and STAT3 in asthmatic AECs. ELISA was used to measure the concentration of IL-5 and IL-6. Furthermore, the targeting relationship between microRNA(miR)-200c-3p and IL6ST was investigated using a luciferase reporter gene assay. Compared with normal human bronchial epithelial cells, HDM-induced AECs had lower expression level of miR-200c-3p, higher mRNA and protein expression levels of IL6ST and an increase in IL-5 and IL-6 concentration. Both MIM and miR-200c-3p mimics suppressed the secretion of IL-5 and L- 6 and promoted the proliferation of HDM-induced AECs. MIM could also upregulate miR-200c-3p and downregulate IL6ST and proteins in the JNK/STAT3 pathway. IL6ST was found to be a downstream target of miR-200c-3p. Inhibition of miR-200c-3p reversed the suppression of asthmatic inflammation by MIM. In summary, MIM upregulated miR-200c-3p expression level to reduce the protein and mRNA expression levels of IL6ST and suppress its downstream JNK/STAT3 signaling pathway, therefore inhibiting the asthmatic inflammation of AECs.
\end{abstract}

Correspondence to: Dr Linliang Hong, Department of Pediatrics, The First Affiliated Hospital of Fujian Medical University, 20 Chazhong Road, Taijiang, Fuzhou, Fujian 350005, P.R. China E-mail: trap2008@163.com

Key words: asthma, house dust mite, microRNA-200c-3p, metabolites of intestinal microflora, JNK/STAT3 signaling pathway

\section{Introduction}

Asthma is a chronic inflammatory disease of the conducting airways and affects up to 334 million individuals globally $(1,2)$. Increasing experimental evidence has suggested that the airway epithelium, the first line of defense against the exposure of airway tissue to an inflammatory reaction, plays a pivotal role in asthma pathogenesis (3). Airway epithelial cell (AEC) inflammation is one of the characteristics of asthma $(4,5)$. Given that AEC injury is involved in asthma pathophysiology, maintaining AEC survival and function may be an effective therapeutic strategy for asthma (6). However, the molecular mechanisms of AEC inflammation in asthma are still unknown.

Micro(mi)RNAs are short endogenous RNAs that are able to regulate gene expression by binding to their target mRNA to alter mRNA degradation and translation (7-9). The majority of asthma-related protein-coding genes have been found to be regulated by miRNAs, suggesting that miRNAs could serve as novel biomarkers of asthma (10-12). Recently, a number of studies has revealed that miR-200b/c played a role in asthma, and a decrease in miR-200b/c may be an underlying cause of the asthmatic phenotype $(13,14)$. However, the regulatory mechanism of miR-200c-3p in asthma requires further investigation.

A diverse microbial community exists in the intestinal environment and mediates the composition of chemical signals and metabolites within the gut (15). The human intestinal microflora, where millions of genes are expressed, regulates numerous host physiological processes, including energy consumption, nutritional homeostasis and immunity $(16,17)$. Notably, a previous study supported the possibility of microbiota-directed therapies for asthma (18). Metabolites of intestinal microflora (MIM) have been reported to inhibit colorectal cancer cell migration by increasing miR-200c expression levels (19). However, the association between MIM and miR-200c-3p remains unclear in asthmatic AECs.

The present study investigated the regulation of miR-200c-3p by MIM in asthmatic inflammation and provided novel insights for the targeted therapy of asthma.

\section{Materials and methods}

Ethical statement. The present study was approved by the Ethics Committee of The First Affiliated Hospital of Fujian 
Medical University. All efforts were taken to minimize the suffering of the animals.

Culture of intestinal microflora and collection of MIM from mice. A total of three healthy male C57BL/c mice (8 weeks old; 20-25 g) were purchased from Hunan SJA Laboratory Animal Co., Ltd. All the animals were housed in a specific pathogen-free environment, with free access to food and water, and were provided with 12-h shifts of light/dark cycles. The intestinal metabolites were obtained from one of the three mice each time. An intestinal microflora suspension was prepared by mixing $0.25 \mathrm{~g}$ fresh mouse feces with $1 \mathrm{ml} 0.9 \%$ $\mathrm{NaCl}$ in a sterile tube. Every $1 \mathrm{ml}$ intestinal microflora suspension was mixed with $9 \mathrm{ml}$ anaerobic medium (Gifu Anaerobic Broth medium; Nissui Pharmaceutical Co., Ltd.) and placed in a culture bag. The culture bag was sealed after the addition of an anaerobic bag and an oxygen indicator and was incubated at $36^{\circ} \mathrm{C}$ for $48 \mathrm{~h}$, during which the culture bag was oscillated at $100 \mathrm{r} / \mathrm{min}$ for the first $24 \mathrm{~h}$ and maintained at a still position for the remaining $24 \mathrm{~h}$. Thereafter, the MIM were collected and centrifuged twice for $15 \mathrm{~min}$ at $10,000 \mathrm{x} \mathrm{g,} 4^{\circ} \mathrm{C}$. The supernatant was preserved and filtered using a 0.22 -um membrane under sterile conditions.

Epithelial cell culture and transfection. The human bronchial epithelial cells (16HBE) were purchased from Cell Resource Center, Peking Union Medical College and cultured according to the manufacturer's instructions. The cells in the logarithmic phase were treated with saline or $400 \mathrm{U} / \mathrm{ml}$ HDMs (Sigma-Aldrich; Merck KGaA) for $24 \mathrm{~h}$ and correspondingly divided into the Control or HDM groups. 16HBE cells, which were pretreated with MIM before HDM induction were assigned to the MIM group. Additional $16 \mathrm{HBE}$ cells, also in the logarithmic growth phase, were transfected with miR-200c-3p mimics (sense, 5'-UAAUACUGCCGG GUAAUGAUGGA-3'; antisense, 5'-UCCAUCAUUACCCGG CAGUAUUA-3'), miR-200c-3p inhibitor (5'-UCCAUCAUU ACCCGGCAGUAUUA-3'), miR-200c-3p mimics negative control (NC; sense, 5'-GUAGCUUAUCCGGAAUCUAAG UC-3'; antisense, 5'-GACUUAGAUUCCGGAUAAGCU AC-3') or miR-200c-3p inhibitor NC (5'-GACUUAGAUUCC GGAUAAGCUAC-3') (Guangzhou RiboBio Co., Ltd.) before HDM induction, and were divided into the following groups: miR-200c-3p mimics, miR-200c-3p inhibitor, NC mimics and $\mathrm{NC}$ inhibitor, respectively. A portion of the cells in the miR-200c-3p inhibitor group or the NC inhibitor group were treated with MIM before HDM induction and correspondingly termed the MIM + miR-200c-3p inhibitor and MIM + NC inhibitor groups. The final concentration of miR-200c-3p mimics, miR-200c-3p inhibitor or NCs in the culture medium was $50 \mathrm{nM}$.

All the transfections were performed using Lipofectamine ${ }^{\circledR}$ 2000 (Invitrogen; Thermo Fisher Scientific, Inc.) according to the manufacturer's instructions. The transfection was sustained at $37^{\circ} \mathrm{C}$ for $4 \mathrm{~h}$. The following experiments were performed $48 \mathrm{~h}$ after the transfection. The transfected cells were suspended in DMEM (10\% FBS) and seeded in 24-well plates at a density of $1 \times 10^{5}$ cells/well and cultured at $37^{\circ} \mathrm{C}$ in humidified incubator with $5 \% \mathrm{CO}_{2}$. Cells pretreated with $20 \mu \mathrm{M}$ SP600125 (JNK inhibitor) for $1 \mathrm{~h}$ before HDM (400 U/ml) stimulation for $24 \mathrm{~h}$ were termed as the SP600125 group, while the cells pretreated with $100 \mu \mathrm{M}$ S3I-201 (STAT3 inhibitor) for $1 \mathrm{~h}$ before HDM stimulation were termed as the S3I-201 group.

MTT assay. After the cells were incubated for 24,48 and 72 h, $20 \mu \mathrm{l}$ MTT solution (5 mg/ml; Merck KGaA) was added to each group and the cells were cultured at $37^{\circ} \mathrm{C}$ with $5 \%$ $\mathrm{CO}_{2}$ for $4 \mathrm{~h}$. Thereafter, the culture medium was discarded and replaced with $150 \mu \mathrm{l}$ dimethyl sulfoxide. The plate was gently shaken for $10 \mathrm{~min}$ to facilitate crystal dissolution. The optical density (OD) of each well was measured at $570 \mathrm{~nm}$ using an enzyme-linked immunometric meter. The MTT curve was created using OD as the ordinate variable and time as the abscissa. The OD was obtained from three independent experiments and each sample was calculated three times and averaged to obtain the average value.

Reverse transcription-quantitative PCR (RT-qPCR). RNA was extracted from $16 \mathrm{HBE}$ cells using $1 \mathrm{ml}$ TRIzol ${ }^{\circledR}$ (Invitrogen; Thermo Fisher Scientific, Inc.) according to the manufacturer's instructions. After quantification by NanoDrop 2000 (Thermo Fisher Scientific, Inc.), the RNA was reverse transcribed into cDNA using One Step PrimeScript RT-PCR Kit (Takara Bio, Inc.). The RT reaction was maintained at $37^{\circ} \mathrm{C}$ for $60 \mathrm{~min}$ and then at $85^{\circ} \mathrm{C}$ for $5 \mathrm{sec}$. qPCR was conducted using a SYBR Green fluorescent qPCR kit (Takara Biotechnology Co., Ltd.) and the CFX96 real-time PCR system (Applied Biosystems; Thermo Fisher Scientific, Inc.). The following thermocycling conditions were used: Initial denaturation at $95^{\circ} \mathrm{C}$ for $10 \mathrm{~min}$, followed by 40 cycles of denaturation at $95^{\circ} \mathrm{C}$ for $10 \mathrm{sec}$, annealing at $60^{\circ} \mathrm{C}$ for $20 \mathrm{sec}$ and extension at $72^{\circ} \mathrm{C}$ for $34 \mathrm{sec}$. The threshold value was selected manually at the lowest point of parallel logarithmic amplification curves, and the $\mathrm{Cq}$ value (threshold cycle) of each reaction was obtained. The data was analyzed using the $2^{-\Delta \Delta \mathrm{Cq}}$ method (20), where $2^{-\Delta \Delta \mathrm{Cq}}$ represents the ratio of gene expression between the experimental and control groups: $\Delta \Delta \mathrm{Cq}=[\mathrm{Cq}$ (target gene) $-\mathrm{Cq}$ (reference gene)] experimental group-[Cq (target gene)-Cq (reference gene)] control group. $\mathrm{Cq}$ refers to the cycle number when the real-time fluorescence intensity of the reaction reaches the threshold and the amplification shows a logarithmic manner. The PCR experiments were repeated three times. The internal references for miRNA and mRNA were U6 and GAPDH, respectively. The primer sequences of each gene are listed in Table I.

Western blot analysis. Cells were washed with PBS and lysed in RIPA buffer containing phenylmethylsulfonyl fluoride (Beyotime Institute of Biotechnology) on ice for $30 \mathrm{~min}$. Afterwards, the cell lysates were centrifuged at $12,000 \mathrm{x} \mathrm{g}$ for $10 \mathrm{~min}$ at $4^{\circ} \mathrm{C}$. The supernatant was then transferred to $0.5-\mathrm{ml}$ centrifuge tubes and stored at $-20^{\circ} \mathrm{C}$ or quantified using a BCA kit. Next, 6X SDS loading buffer was added to the tubes for protein denaturation at $100^{\circ} \mathrm{C}$ for $5 \mathrm{~min}$. The proteins (40 $\mu$ g per lane) were then separated using 12\% SDS-PAGE and transferred onto a PVDF membrane with pre-cooled transfer buffer at $4^{\circ} \mathrm{C}$ for $1.5 \mathrm{~h}$. The membrane was blocked with $5 \%$ skimmed milk in TBS-0.05\% Tween-20 (TBST) buffer for $1 \mathrm{~h}$. TBST-diluted primary antibodies were then incubated with the membrane overnight at $4^{\circ} \mathrm{C}$. The membrane was 
Table I. Primer sequences used for reverse transcription-quantitative PCR.

\begin{tabular}{ll}
\hline Name & \multicolumn{1}{c}{ Sequence (5'-3') } \\
\hline miR-200c F & GGTAATACTGCCGGGTAAT \\
miR-200c R & CAGTGCGTGTCGTGGAGT \\
U6 F & CTCGCTTCGGCAGCACATATACT \\
U6 R & ACGCTTCACGAATTTGCGTGTC \\
IL6ST F & GTGTTTAGGATTCGCTGTATGA \\
IL6ST R & CTGTAGCCTTGAGTATGGGATG \\
GAPDH F & GTCAGTGGTGGACCTGACCT \\
GAPDH R & TGCTGTAGCCAAATTCGTTG \\
\hline
\end{tabular}

F, forward; R, reverse; miR, microRNA.

then washed with TBST 3 times each for $10 \mathrm{~min}$. Thereafter, the secondary goat anti-rabbit IgG antibody (1:5,000; cat. no. CW0103S; Beijing ComWin Biotech Co., Ltd.) was incubated with the membrane for $2 \mathrm{~h}$ at room temperature. After the incubation, the membrane was washed with TBST. The protein expression levels of IL6ST, phosphorylated (p)-STAT3, STAT3, p-JNK and JNK were detected by densitometry using an enhanced ECL chemiluminescence substrate kit (cat. no. CW0049; Beijing ComWin Biotech Co., Ltd.) and ImageJ software (version 1.46; National Institutes of Health). The following primary antibodies were used: Anti-IL6ST (1:1,000; cat. no. ab202850; Abcam), anti-STAT3 (1:1,000; cat. no. 12640S; Cell Signaling Technology, Inc.), anti-p-STAT3 (1:1,000; cat. no. 9145T; Cell Signaling Technology, Inc.), anti-JNK (1:1,000; cat. no. ab179461; Abcam), and anti-p-JNK (1:1,000; cat. no. ab124956; Abcam). Anti-GAPDH antibody (1:500; 5174T; Cell Signaling Technology, Inc.) was used as the internal reference.

ELISA. The supernatant of each group was collected after the $16 \mathrm{HBE}$ cells were stimulated with HDM for $24 \mathrm{~h}$. ELISA kits were used to determine the IL-5 (Human IL-5 Quantikine ELISA Kit; cat. no. D5000B; R\&D Systems, Inc.) and IL-6 (Human IL-6 Quantikine ELISA kit; cat. no. D6050; R\&D Systems, Inc.) contents in the supernatant. All the experiments were performed according to the manufacturer's instructions.

Dual-luciferase reporter gene assay. The target binding sites between IL6ST and miR-200c-3p were predicted by the online tool StarBase v3.0 (http://starbase.sysu.edu.cn/). According to the prediction, wild-type (WT) and mutant-type (MT) sequences of the binding sites between IL6ST and miR-200c-3p were designed. MT and WT fragments of IL6ST 3'-untranslated region (UTR) were cloned and ligated into a luciferase vector (pGL3-Promoter; Promega Corporation) and co-transfected with miR-200c-3p mimics, miR-200c-3p inhibitors or their respective NCs (sequences as aforementioned) into $16 \mathrm{HBE}$ cells, and the groups were termed as: $\mathrm{MT}+$ mimics group, $\mathrm{MT}+\mathrm{NC}$ mimics group, $\mathrm{MT}+$ inhibitors group, $\mathrm{MT}+\mathrm{NC}$ inhibitors group, $\mathrm{WT}+$ mimics group, $\mathrm{WT}+$ NC mimics group, WT + inhibitors group, or WT + NC inhibitors group. In addition, pRL-TK vector (Renilla luciferase) was co-transfected into the cells as internal reference. After transfection for $48 \mathrm{~h}$, the luciferase activity of each group was measured using a dual-luciferase reporter assay kit (Generay Biotech Co., Ltd.). The ratio of firefly luciferase activity to Renilla luciferase activity was the relative luciferase activity. The relative luciferase activity of the control groups was set to 1 , and the data of the other groups were represented as the ratio to the control group.

Statistical analysis. Statistical analysis was performed using the SPSS v17.0 (SPSS, Inc.) and GraphPad Prism v6.0 (GraphPad software, Inc.). Data are presented as the mean \pm standard deviation, and all experiments were repeated three times. An unpaired Student's t-test was used to compare two groups, while one-way analysis of variance was used for comparisons among multiple groups, followed by a Tukey's post hoc test. $\mathrm{P}<0.05$ indicates a statistically significant difference.

\section{Results}

Low miR-200c-3p expression and high IL6ST expression in HDM-induced cells. First, 200, 400 and $800 \mathrm{U} / \mathrm{ml}$ HDM was used to treat the $16 \mathrm{HBE}$ cells in vitro for $24 \mathrm{~h}$ and the results from ELISA showed that the concentrations of the inflammatory mediators, IL-5 and IL-6, were elevated in contrast to those in the Control group, indicating that HDM successfully induced an inflammatory response in the $16 \mathrm{HBE}$ cells $(\mathrm{P}<0.05$; Fig. 1A and $\mathrm{B})$. In addition, the secretion of IL-5 and IL-6 levelled off at $800 \mathrm{U} / \mathrm{ml} \mathrm{HDM} \mathrm{(Fig.} \mathrm{1A} \mathrm{and} \mathrm{B).} \mathrm{Thus,}$ $400 \mathrm{U} / \mathrm{ml} \mathrm{HDM}$ was used for the following experiments.

Next, the mRNA expression levels of miR-200c-3p and IL6ST in the HDM-induced cells was determined. RT-qPCR showed that compared with that in the control cells, the mRNA expression level of miR-200c-3p was decreased in the HDM-induced cells $(\mathrm{P}<0.05$; Fig. $1 \mathrm{C})$, indicating that HDM suppressed miR-200c-3p mRNA expression level. In addition, the mRNA and protein expression level of IL6ST in the HDM-induced cells was found to be higher compared with that in the Control group ( $\mathrm{P}<0.05$; Fig. $1 \mathrm{D}$ and $\mathrm{E}$, respectively). The aforementioned findings indicated that miR-200c-3p and IL6ST could be involved in HDM-induced inflammation of the $16 \mathrm{HBE}$ cells.

MIM increases miR-200c-3p mRNA expression and decreases IL6ST mRNA and protein expression levels to inhibit airway inflammation in the epithelial cells. Next, the 16HBE cells were pretreated with MIM and induced with HDM for $24 \mathrm{~h}$ to determine the effect of MIM on the mRNA expression level of miR-200c-3p and IL6ST. As shown in Fig. 2A, the MIM group showed enhanced cell viability compared with that in the HDM group $(\mathrm{P}<0.05)$. In addition, compared with that in the HDM group, the MIM group showed increased miR-200c-3p mRNA expression levels $(\mathrm{P}<0.05$; Fig. 2B) and decreased IL6ST mRNA and protein expression levels $(\mathrm{P}<0.05$; Fig. $2 \mathrm{C}$ and $\mathrm{D}$, respectively), and decreased concentrations of IL-5 and IL-6 $(\mathrm{P}<0.05$; Fig. 2E).

miR-200c-3p blocks HDM-induced elevation of IL-5 and IL-6 levels by regulating IL6ST expression. To investigate 
A

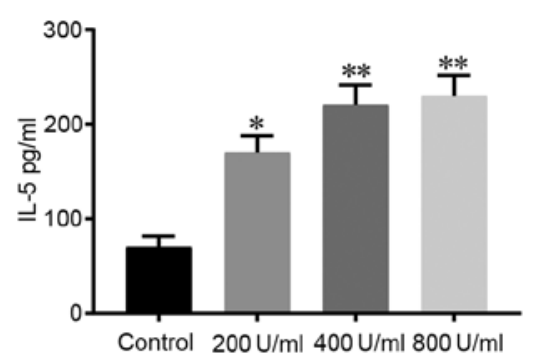

D

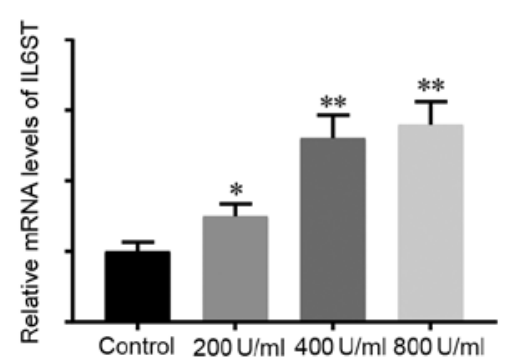

B

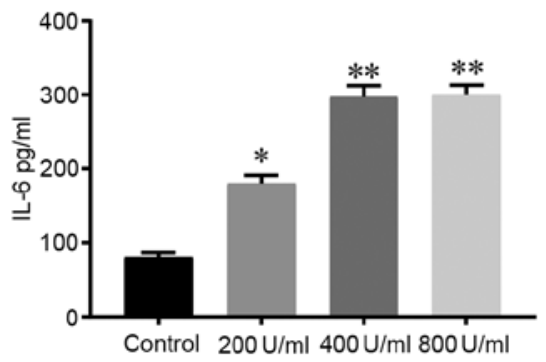

E
C

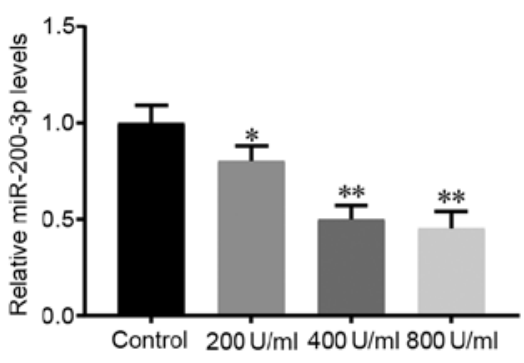

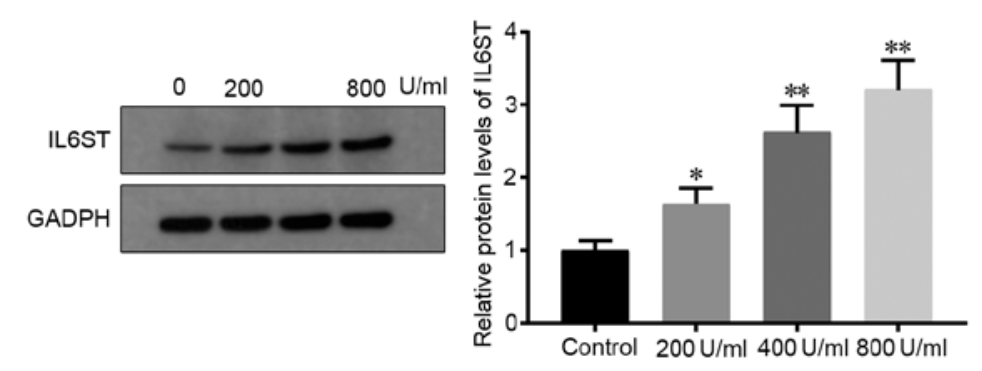

Figure 1. Dysregulation of miR-200c-3p and IL6ST in the HDM-induced cells. After the 16HBE cells were treated with 200, 400 and 800 U/ml HDM for $24 \mathrm{~h}$, the concentration of (A) IL-5 and (B) IL-6 was measured using ELISA. (C) The mRNA expression level of miR-200c-3p was measured using RT-qPCR. (D) RT-qPCR and (E) western blot analysis was used to determine the mRNA and protein expression levels of IL6ST, respectively. "P<0.05, ${ }^{* *} \mathrm{P}<0.01$, vs. Control group. HDM, house dust mite; miR, microRNA; RT-qPCR, reverse transcription-quantitative PCR.

A

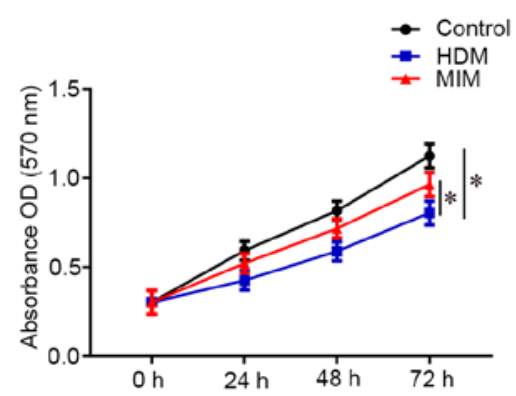

B

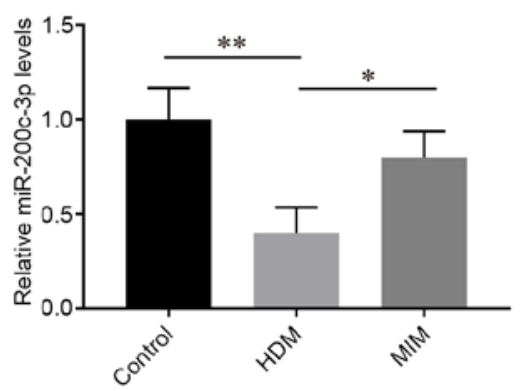

C

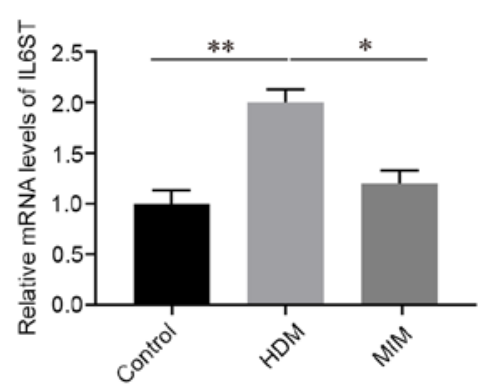

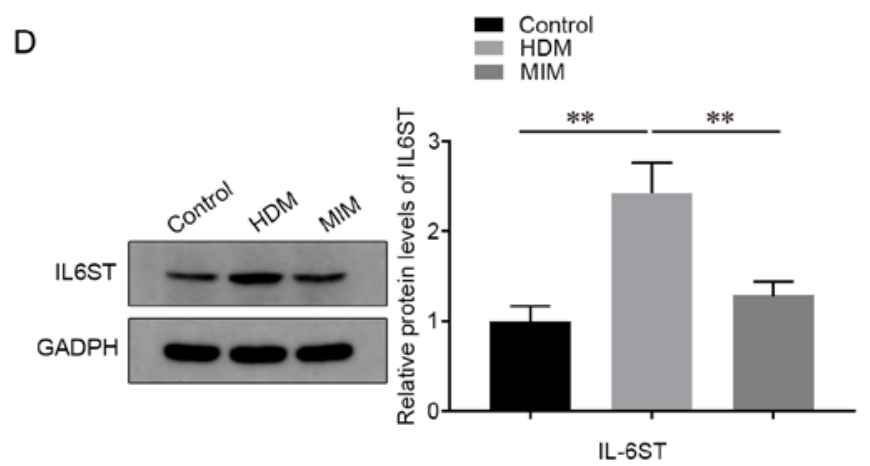

E

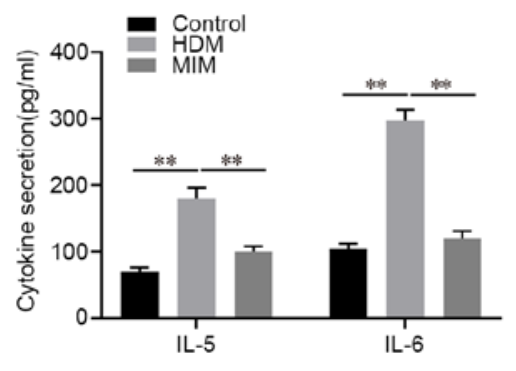

Figure 2. MIM increases miR-200c-3p mRNA expression levels and decreases IL6ST mRNA and protein expression levels in the HDM-induced cells. (A) After the 16HBE cells were pretreated with MIM, they were treated with HDM for $24 \mathrm{~h}$ and cell viability was measured using a MTT assay. (B) The mRNA expression level of miR-200c-3p was detected using RT-qPCR. The (C) mRNA and (D) protein expression levels of IL6ST were detected using RT-qPCR and western blot analysis, respectively. (E) ELISA was used to measure IL-5 and IL- 6 concentrations. ${ }^{*} \mathrm{P}<0.05,{ }^{* *} \mathrm{P}<0.01$. MIM, metabolites of intestinal microflora; HDM, house dust mite; miR, microRNA; RT-qPCR, reverse transcription-quantitative PCR; OD, optical density.

the effect of miR-200c-3p on the biological activities of the HDM-induced cells, the miR-200c-3p mimics or -miR-200c-3p inhibitor were transfected into the $16 \mathrm{HBE}$ cells, after which the cells were stimulated by HDM for $24 \mathrm{~h}$. RT-qPCR results 
A

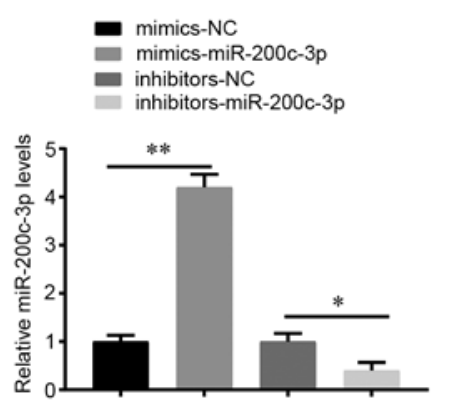

B

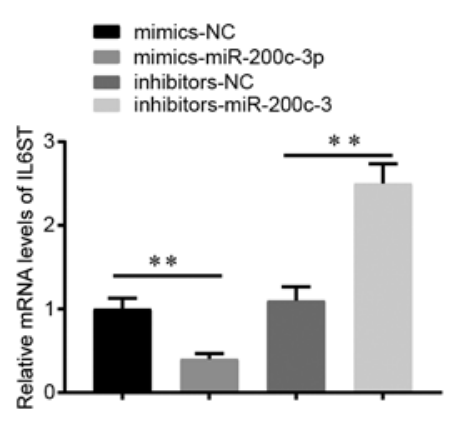

C

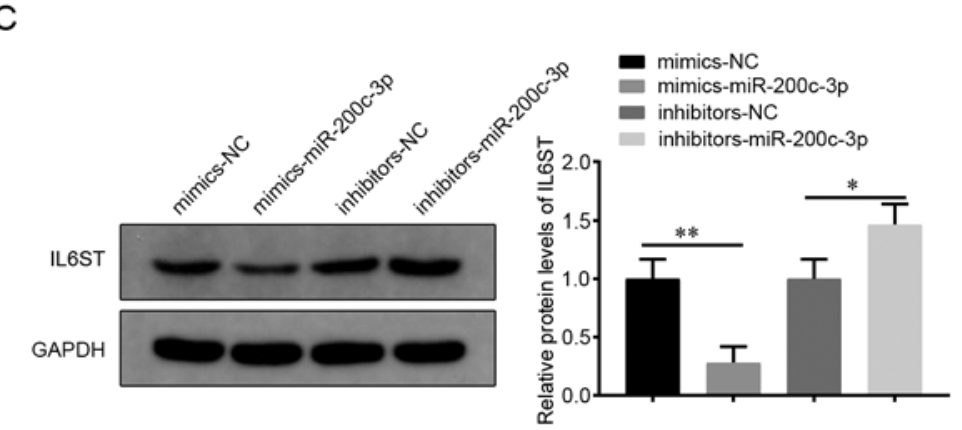

D

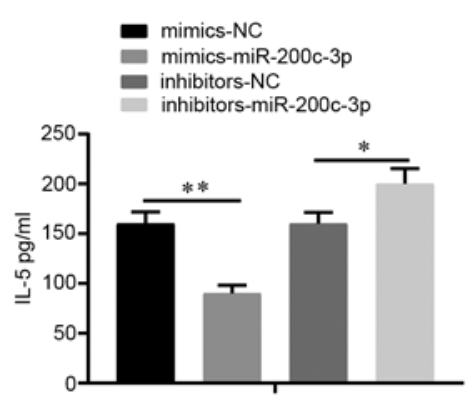

E

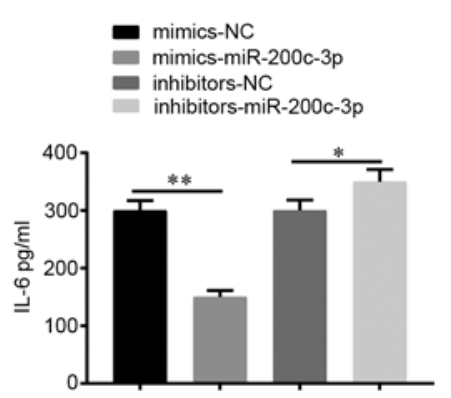

Figure 3. miR-200c-3p blocks HDM-induced elevation of IL-5 and IL-6 levels by mediating mRNA and protein IL6ST expression. The 16HBE cells were transfected with miR-200c-3p mimics or miR-200c-3p inhibitors then treated with HDM for $24 \mathrm{~h}$. (A) The expression level of miR-200c-3p was determined using RT-qPCR. (B) RT-qPCR and (C) western blot analysis was used to examine the mRNA and protein expression levels of IL6ST, respectively. The concentration of (D) IL-5 and (E) IL-6 in each group was detected using ELISA. "P<0.05, ${ }^{* *} \mathrm{P}<0.01$. HDM, house dust mite; miR, microRNA; RT-qPCR, reverse transcription-quantitative PCR; NC, negative control.

showed that the miR-200c-3p mimics group had an increased miR-200c-3p mRNA expression level (vs. NC mimics) and the miR-200c-3p inhibitor group had a decreased miR-200c-3p mRNA expression level (vs. NC inhibitor group) ( $\mathrm{P}<0.05$; Fig. 3A), indicating effective miR-200c-3p overexpression or silencing in the HDM-induced cells.

Compared with that in the NC mimics group, the miR-200c-3p mimics group had lower mRNA and protein IL6ST expression levels ( $\mathrm{P}<0.01$; Fig. 3B and $\mathrm{C})$ and suppressed the secretion of IL-5 and IL-6 ( $\mathrm{P}<0.01$; Fig. 3D and E, respectively). The opposite effect was found for the mRNA and protein expression levels of IL6ST $(\mathrm{P}<0.05$; Fig. 3B and C) and the concentration of IL-5 and IL-6 in the miR-200c-3p inhibitor group compared with that in the NC inhibitor group $(\mathrm{P}<0.05$; Fig. 3D and E). The aforementioned results indicated that miR-200c-3p could inhibit HDM-induced inflammation in $16 \mathrm{HBE}$ cells.
miR-200c-3p inhibitor reverses the suppression of $H D M$-induced inflammation by MIM. A rescue experiment was designed to further validate the effect of miR-200c-3p on HDM-induced inflammation. Compared with that in the MIM + NC inhibitor group, the miR-200c-3p inhibitor reduced the mRNA expression level of miR-200c-3p in HDM-induced 16HBE cells treated with MIM $(\mathrm{P}<0.05$; Fig. 4A) and increased the secretion of IL-5 and IL-6 (P<0.01; Fig. 4B). Furthermore, the mRNA and protein expression levels of IL6ST were also increased in the MIM + miR-200c-3p inhibitor group compared with that in the MIM + NC inhibitor group ( $\mathrm{P}<0.01$; Fig. $4 \mathrm{C}$ and $\mathrm{D})$. Collectively, these data showed that the miR-200c-3p inhibitor reversed the suppression of HDM-induced inflammation by MIM in AECs.

IL6ST is a functional target of miR-200c-3p. Based on the prediction results from the StarBase v3.0 database, the putative 
A

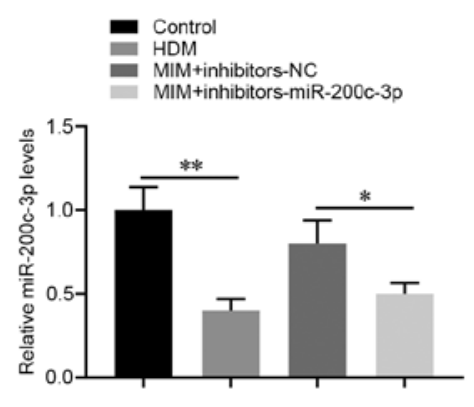

C

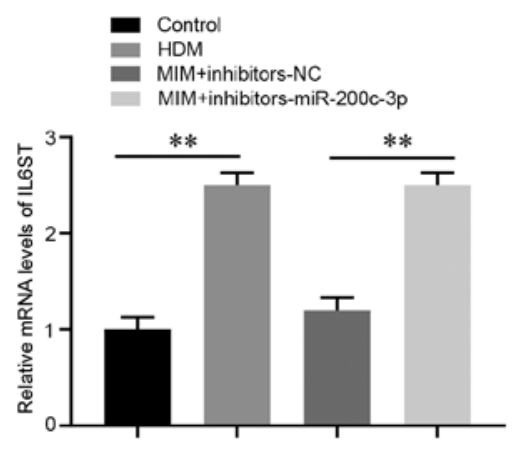

B

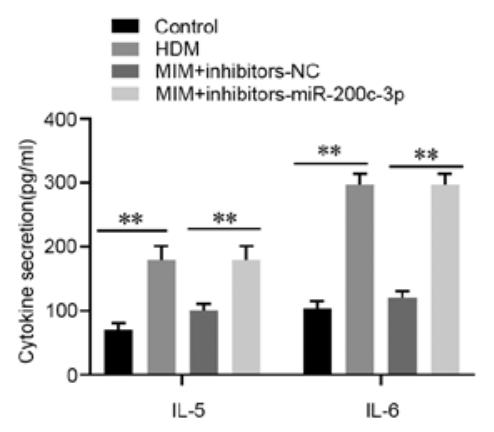

D

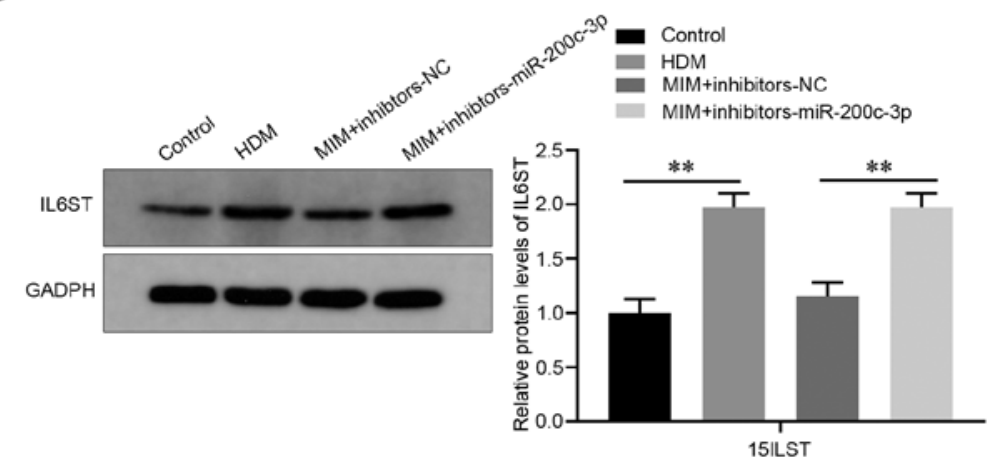

Figure 4. miR-200c-3p inhibitors reverse the protective effect of MIM on HDM-induced airway epithelial cells. The 16HBE cells were transfected with miR-192-5p inhibitors or NC inhibitors and then treated with metabolites of intestinal microflora before HDM induction. (A) RT-qPCR was used to measure the expression level of miR-200c-3p. (B) The concentrations of IL-5 and IL-6 were detected using ELISA. (C) RT-qPCR and (D) Western blot assays examined the mRNA and protein expression level of IL6ST, respectively. ${ }^{*} \mathrm{P}<0.05,{ }^{* *} \mathrm{P}<0.01$. HDM, house dust mite; miR, microRNA; RT-qPCR, reverse transcriptionquantitative PCR; NC, negative control.

A

\author{
MUT IL6ST:5' CaCUUCGAGCACUGUCGUCAUAAC 3' \\ WT IL6ST:5' caCUUCGAGCACUGUCCAGUAUUc 3' \\ | || ||: ||||||| \\ miR-200c-3p :3' agGUAG-UAAUGGGCCGUCAUAAu 5'
}

B
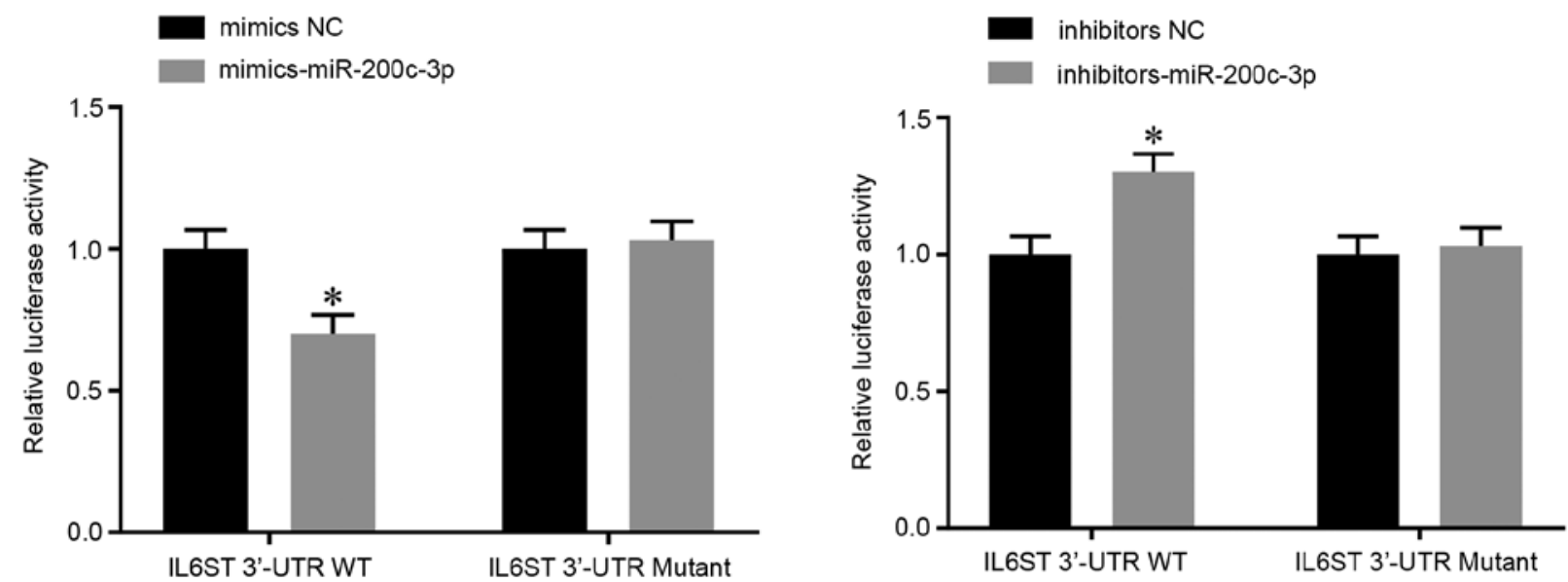

Figure 5. IL6ST is a target gene of miR-200c-3p. (A) The binding sites between IL6ST and miR-200c-3p were predicted using the StarBase database. (B) A dual-luciferase reporter assay was used to analyze the binding between IL6ST and miR-200c-3p. "P<0.05, compared with the WT + NC group. WT, wild-type; UTR, untranslated region; miR, microRNA; NC, negative control; MUT, mutant-type. 

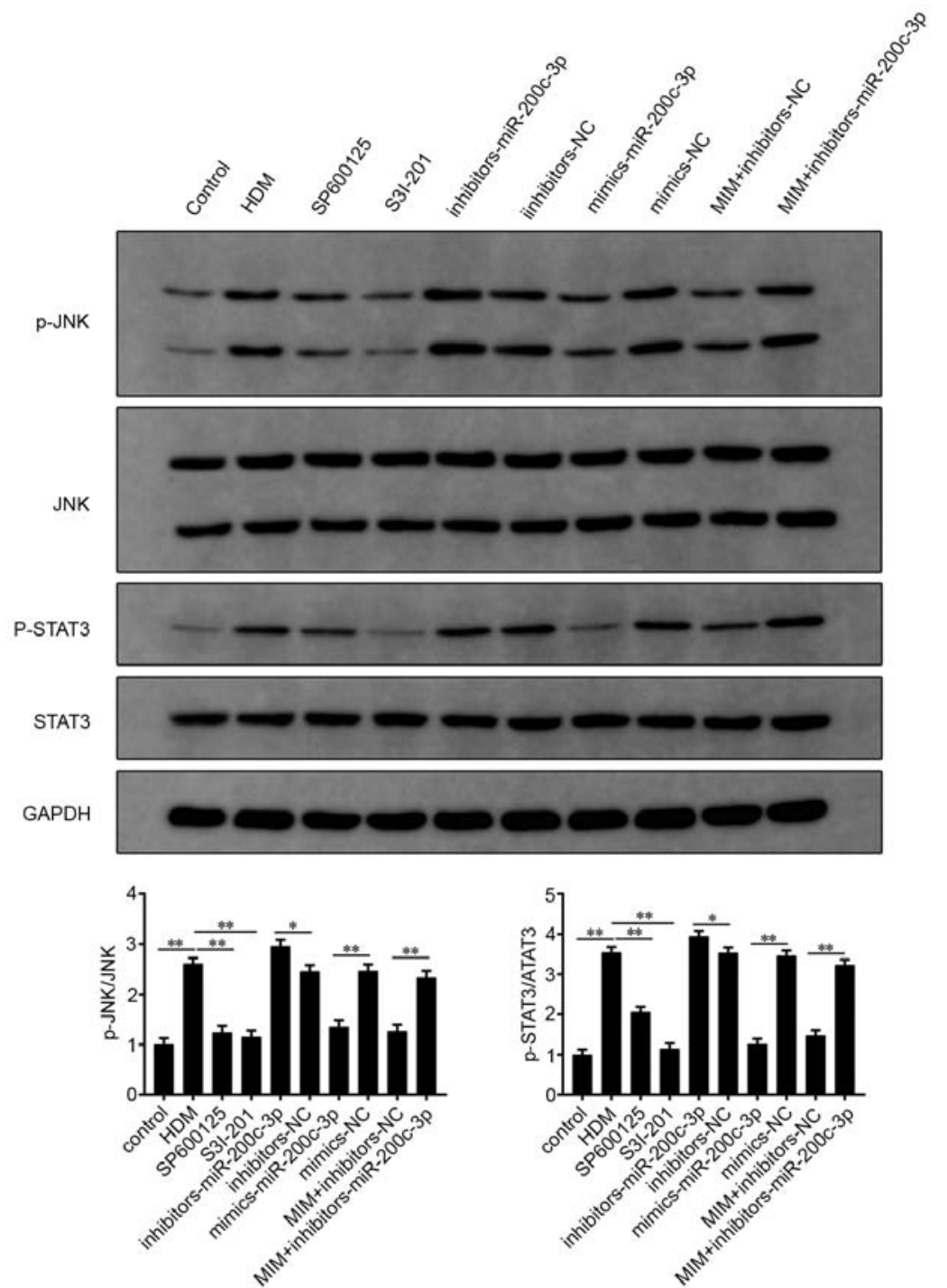

Figure 6. MIM showed anti-inflammatory effects on HDM-induced airway epithelial cells via the JNK/STAT3 signaling pathway. The 16HBE cells were pretreated with $20 \mu \mathrm{M} \mathrm{SP} 600125$ or $100 \mu \mathrm{M} \mathrm{S3I}-201$ for $1 \mathrm{~h}$, followed by HDM (400 U/ml) stimulation for $24 \mathrm{~h}$. The $16 \mathrm{HBE}$ cells were also transfected with miR-200c-3p mimics or miR-200c-3p inhibitors and treated with HDM (400 U/ml) for $24 \mathrm{~h}$. The 16HBE cells were also transfected with miR-200c-3p inhibitors and treated with MIM followed by HDM stimulation $(400 \mathrm{U} / \mathrm{ml})$ for $24 \mathrm{~h}$. The protein expression levels of total and phosphorylated JNK and STAT3 were detected using western blot analysis. ${ }^{*} \mathrm{P}<0.05,{ }^{* *} \mathrm{P}<0.01$. MIM, metabolites of intestinal microflora; HDM, house dust mite; miR, microRNA; NC, negative control.

binding site of miR-200c-3p was located on the 3'-UTR of IL6ST (Fig. 5A). The results of the luciferase reporter gene assay showed that compared with that in the WT + NC group, the luciferase activity in the $\mathrm{WT}+$ mimics group was markedly inhibited, whereas the WT + inhibitor group showed increased luciferase activity $(\mathrm{P}<0.05$; Fig. 5B). In addition, no visible difference in luciferase activity was observed between the MT + mimics group and MT $+\mathrm{NC}$ group or between the $\mathrm{MT}+$ inhibitors group and MT + NC group. These results suggested that miR-200c-3p targeted the 3'-UTR of IL6ST to inhibit the expression level of IL6ST in asthmatic AECs.

MIM suppress inflammation in asthmatic AECs by regulating the JNK/STAT3 signaling pathway. As shown in Fig. 6, p-JNK and p-STAT3 expression levels were significantly higher in the HDM-induced $16 \mathrm{HBE}$ cells $(\mathrm{P}<0.01$; HDM group vs. control group). The addition of the JNK inhibitor, SP600125 and the STAT3 inhibitor, S3I-201, alone, suppressed HDM-induced
JNK and STAT3 phosphorylation $(\mathrm{P}<0.01$; SP600125 or S3I-201 group vs. HDM group). Furthermore, the miR-200c-3p mimics group had decreased $\mathrm{p}-\mathrm{JNK}$ and $\mathrm{p}$-STAT3 protein expression levels, whereas the miR-200c-3p inhibitor group had elevated $\mathrm{p}$-JNK and $\mathrm{p}$-STAT3 protein expression levels compared with that in their respective NC groups $(\mathrm{P}<0.05)$. In addition, the expression levels of p-JNK and p-STAT3 in the $\mathrm{MIM}+\mathrm{miR}-200 \mathrm{c}-3 \mathrm{p}$ inhibitor group were significantly higher compared with that in the MIM + NC inhibitor group $(\mathrm{P}<0.01)$. The aforementioned results indicated the involvement of the JNK/STAT3 signaling pathway in MIM-mediated inflammation in asthmatic AECs.

\section{Discussion}

The prevalence of asthma has been increasing over the last few decades and this has been accompanied by a substantial economic burden (21). Therefore, there is an urgent 
requirement to improve the precise pathophysiology of asthma and achieve an optimal therapeutic effect on this disease. In the present study, significantly lower miR-200c-3p mRNA and higher IL6ST mRNA and protein expression levels were detected in HDM-induced AECs. Further analyses showed that MIM could upregulate miR-200c-3p mRNA expression levels to inhibit the IL6ST/JNK/STAT3 signaling pathway; thus, decreasing inflammation in HDM-induced AECs.

The important contribution of the intestinal microflora to human health has been described in previous literature. For example, the intestinal microflora plays an irreplaceable role in promoting or preventing atherosclerotic cardiovascular disease (22). The intestinal microflora has also been associated in the development of autism and mood disorders (23). Recently, the significance of miRNAs in intestinal microflora-mediated host physiology has been elucidated (24-27). The findings of the present study revealed that MIM treatment enhanced the viability and decreased the inflammatory responses of asthmatic AECs. Notably, MIM exerted this protection by upregulating the mRNA expression level of miR-200c-3p.

Several studies have reported that miRNAs modulate inflammatory responses and airway remodeling in asthma $(28,29)$. miR-200c-3p has been identified as a tumor inhibitor in numerous types of human cancer, including breast cancer, prostate cancer and clear cell renal cell carcinoma $(30,31)$. However, the potential role of miR-200c-3p has not been investigated in asthma. In the present study, decreased mRNA expression of miR-200c-3p and increased mRNA and protein expression level of IL6ST was found in the HDM-induced AECs. The transfection of miR-200c-3p mimics decreased the mRNA and protein expression level of IL6ST, as well as decreasing the secretion of 2 inflammatory factors. Based on online software prediction, it was found that miR-200c-3p could target IL6ST; thus, inhibiting the transcription and translation of IL6ST. A rescue experiment was designed to understand the involvement of miR-200c-3p in the MIM-mediated suppression of the asthmatic inflammation of AECs. The results showed that the miR-200c-3p inhibitor reversed the suppression of HDM-induced AEC inflammation by MIM. Therefore, MIM could upregulate miR-200c-3p to reduce IL6ST-dependent inflammation in AECs.

However, the accurate mechanism by which miR-200c-3p mediated asthmatic inflammation was unclear; thus, the functional mechanism of miR-200c-3p was further investigated. A previous study has shown that activated signaling pathways, including the JNK/STAT3 signaling pathway, were associated with lung cancer cell survival, migration, inflammation and proliferation (32). The current study revealed that the protein expression levels of p-JNK and p-STAT3 were significantly increased in the HDM-induced AECs. Notably, miR-200c-3p could downregulate the phosphorylation levels of JNK and STAT3 and this effect was enhanced when cells were treated with MIM. Previous research suggested that the JNK/STAT3 signaling pathway was associated with apoptosis and participated in the inflammation of myocardial injury (33). Overall, MIM could decrease the phosphorylation of JNK and STAT by promoting miR-200c-3p, thereby enhancing the proliferation of asthmatic AECs.

In summary, the present study uncovered the effect of MIM in asthma and the underlying molecular mechanism and the findings suggested that MIM suppressed AEC inflammation, which can occur in asthma, indicating the possibility of MIM treatment as a novel therapy for asthma. MIM exerted therapeutic effects by regulating the miR-200c-3p/IL6ST axis and the JNK/STAT3 signaling pathway. Nevertheless, there are limitations to the HDM-induced asthma cell models, and there is a lack of animal and clinical experiments to support the results of the cell experiments. In addition, which component plays a role in intestinal metabolites also requires further investigated.

\section{Acknowledgements}

Not applicable.

\section{Funding}

This research was funded by a grant from Startup Fund for Scientific Research of Fujian Medical University (grant no. 2017XQ1079).

\section{Availability of data and materials}

The datasets used and/or analyzed during the current study are available from the corresponding author on reasonable request.

\section{Authors' contributions}

LH conceived the ideas, designed the experiments and supervised the study. $\mathrm{HH}$ and $\mathrm{BW}$ performed the experiments. $\mathrm{LH}$, $\mathrm{HH}$ and $\mathrm{BW}$ analyzed the data. $\mathrm{LH}$ and $\mathrm{BW}$ provided critical materials. $\mathrm{HH}$ and $\mathrm{BW}$ wrote the manuscript. $\mathrm{LH}, \mathrm{HH}$ and $\mathrm{BW}$ confirm the authenticity of all the raw data. All authors have read and approved the final version of the manuscript.

\section{Ethics approval and consent to participant}

The present study was approved by the ethical committee of First Affiliated Hospital of Fujian Medical University.

\section{Patient consent for publication}

Not applicable.

\section{Competing interests}

The authors declare that they have no competing interests.

\section{References}

1. Lambrecht BN and Hammad H: The immunology of asthma. Nat Immunol 16: 45-56, 2015.

2. Papi A, Brightling C, Pedersen SE and Reddel HK: Asthma. Lancet 391: 783-800, 2018.

3. Gon Y and Hashimoto S: Role of airway epithelial barrier dysfunction in pathogenesis of asthma. Allergol Int 67: 12-17, 2018.

4. Mertens TCJ, Karmouty-Quintana H, Taube C and Hiemstra PS: Use of airway epithelial cell culture to unravel the pathogenesis and study treatment in obstructive airway diseases. Pulm Pharmacol Ther 45: 101-113, 2017.

5. Mitchell PD and O'Byrne PM: Epithelial-derived cytokines in asthma. Chest 151: 1338-1344, 2017. 
6. Zhang H, Sun Y, Rong W, Fan L, Cai Y, Qu Q, Gao Y and Zhao H: miR-221 participates in the airway epithelial cells injury in asthma via targeting SIRT1. Exp Lung Res 44: 272-279, 2018.

7. Kho AT, McGeachie MJ, Moore KG, Sylvia JM, Weiss ST and Tantisira KG: Circulating microRNAs and prediction of asthma exacerbation in childhood asthma. Respir Res 19: 128, 2018.

8. Lam JK, Chow MY, Zhang Y and Leung SW: siRNA versus miRNA as therapeutics for gene silencing. Mol Ther Nucleic Acids 4: e252, 2015

9. Ong J, Timens W, Rajendran V, Algra A, Spira A, Lenburg ME, Campbell JD, van den Berge M, Postma DS, van den Berg A, et al: Identification of transforming growth factor-beta-regulated microRNAs and the microRNA-targetomes in primary lung fibroblasts. PLoS One 12: e0183815, 2017.

10. Kivihall A, Aab A, Soja J, Sładek K, Sanak M, Altraja A, Jakiela B, Bochenek G and Rebane A: Reduced expression of miR-146a in human bronchial epithelial cells alters neutrophil migration. Clin Transl Allergy 9: 62, 2019.

11. Liu Z, Zhang XH, Callejas-Diaz B and Mullol J: MicroRNA in United airway diseases. Int J Mol Sci 17: 716, 2016.

12. Svitich OA, Sobolev VV, Gankovskaya LV, Zhigalkina PV and Zverev VV: The role of regulatory RNAs (miRNAs) in asthma. Allergol Immunopathol (Madr) 46: 201-205, 2018.

13. Taka S, Tzani-Tzanopoulou P, Wanstall H and Papadopoulos NG: MicroRNAs in asthma and respiratory infections: Identifying common pathways. Allergy Asthma Immunol Res 12: 4-23, 2020.

14. Tang X, Wu F, Fan J, Jin Y, Wang J and Yang G: Posttranscriptional regulation of interleukin-33 expression by MicroRNA-200 in bronchial asthma. Mol Ther 26: 1808-1817, 2018.

15. Lustri BC, Sperandio V and Moreira CG: Bacterial chat: Intestinal metabolites and signals in host-microbiota-pathogen interactions. Infect Immun 85: e00476-17, 2017.

16. Tuddenham S and Sears CL: The intestinal microbiome and health. Curr Opin Infect Dis 28: 464-470, 2015.

17. Wang G, Huang S, Wang Y, Cai S, Yu H, Liu H, Zeng X, Zhang G and Qiao S: Bridging intestinal immunity and gut microbiota by metabolites. Cell Mol Life Sci 76: 3917-3937, 2019.

18. Ver Heul A, Planer J and Kau AL: The human microbiota and asthma. Clin Rev Allergy Immunol 57: 350-363, 2019.

19. Xu Z, Tao J, Chen P, Chen L, Sharma S, Wang G and Dong Q: Sodium butyrate inhibits colorectal cancer cell migration by downregulating Bmi-1 through enhanced miR-200c expression. Mol Nutr Food Res 62: e1700844, 2018

20. Livak KJ and Schmittgen TD: Analysis of relative gene expression data using real-time quantitative PCR and the 2(-Delta Delta C(T)) method. Methods 25: 402-408, 2001.

21. Choi JY, Lee HY, Hur J, Kim KH, Kang JY, Rhee CK and Lee SY: TRPV1 blocking alleviates airway inflammation and remodeling in a chronic asthma murine model. Allergy Asthma Immunol Res 10: 216-224, 2018.
22. Jie Z, Xia H, Zhong SL, Feng Q, Li S, Liang S, Zhong H, Liu Z, Gao Y, Zhao H, et al: The gut microbiome in atherosclerotic cardiovascular disease. Nat Commun 8: 845, 2017

23. Mangiola F, Ianiro G, Franceschi F, Fagiuoli S, Gasbarrini G and Gasbarrini A: Gut microbiota in autism and mood disorders. World J Gastroenterol 22: 361-368, 2016.

24. Dong J, Tai JW and Lu LF: miRNA-microbiota interaction in gut homeostasis and colorectal cancer. Trends Cancer 5: 666-669, 2019.

25. Liu S, da Cunha AP, Rezende RM, Cialic R, Wei Z, Bry L, Comstock LE, Gandhi R and Weiner HL: The host shapes the gut microbiota via fecal MicroRNA. Cell Host Microbe 19: 32-43, 2016.

26. Teng Y, Ren Y, Sayed M, Hu X, Lei C, Kumar A, Hutchins E, Mu J, Deng Z, Luo C, et al: Plant-derived exosomal MicroRNAs shape the gut microbiota. Cell Host Microbe 24: 637-652.e8, 2018.

27. Chang CS and Kao CY: Current understanding of the gut microbiota shaping mechanisms. J Biomed Sci 26: 59, 2019.

28. Bartel S, Schulz N, Alessandrini F, Schamberger AC, Pagel P, Theis FJ, Milger K, Noessner E, Stick SM, Kicic A, et al: Pulmonary microRNA profiles identify involvement of Creb1 and Sec1413 in bronchial epithelial changes in allergic asthma. Sci Rep 7: 46026, 2017.

29. Martinez-Nunez RT, Rupani H, Platé M, Niranjan $M$, Chambers RC, Howarth PH and Sanchez-Elsner T: Genome-wide posttranscriptional dysregulation by MicroRNAs in human asthma as revealed by frac-seq. J Immunol 201: 251-263, 2018.

30. Danarto R, Astuti I, Umbas R and Haryana SM: Urine miR-21-5p and miR-200c-3p as potential non-invasive biomarkers in patients with prostate cancer. Turk J Urol 46: 26-30, 2019.

31. Maolakuerban N, Azhati B, Tusong H, Abula A, Yasheng A and Xireyazidan A: MiR-200c-3p inhibits cell migration and invasion of clear cell renal cell carcinoma via regulating SLC6A1. Cancer Biol Ther 19: 282-291, 2018.

32. Bowman B: FADD and its phosphorylation mediate mitogenic signaling in mutant Kras tumors, 2015.

33. Fan S, Sun JB, Li R, Song X and Li J: Lycopene protects myocardial ischemia injury through anti-apoptosis and anti-oxidative stress. Eur Rev Med Pharmacol Sci 23: 3096-3104, 2019.

This work is licensed under a Creative Commons Attribution-NonCommercial-NoDerivatives 4.0 International (CC BY-NC-ND 4.0) License. 